\title{
Construction of a Bio-economic Model to Estimate the Feasibility and Cost of Achieving Water Quality Targets in the Burnett-Mary Region, Queensland
}

\author{
Craig Beverly ${ }^{1}$, Anna Roberts ${ }^{2}$, Geoff Park $^{2}$, Fred Bennett ${ }^{3}$, and Graeme Doole ${ }^{4}$ \\ ${ }^{1}$ Agriculture Research: Department of Environment and Primary Industries, \\ Rutherglen, Australia \\ ${ }^{2}$ Natural Decisions, Pty Ltd, VIC, Australia \\ ${ }^{3}$ Burnett Mary Regional Group Ltd, Bundaberg, QLD, Australia \\ ${ }^{4}$ Centre for Environmental Economics and Policy, \\ University of Western Australia, WA \\ craig.beverly@depi.vic.gov.au
}

\begin{abstract}
The aim of this study was to develop a bio-economic model to estimate the feasibility and net profit (or net costs)s of achieving set water quality targets (sediment, nitrogen and phosphorus load reductions) in the Burnett Mary region of northern Queensland, Australia to with the aim of protecting the southern portion of the Great Barrier Reef (GBR). Two sets of targets were evaluated - Reef Plan Targets (RPTs) which are the currently formally agreed targets, and more ambitious Ecologically Relevant Targets (ERTs) which current science suggests might be needed to better protect the values of the GBR. This paper describes the construct of a bio-economic optimisation framework which has been used to underpin a Water Quality Improvement Plan (WQIP) for the Burnett Mary region. The bioeconomic model incorporates the available science developed from paddock and catchment scale biophysical model results and farm economic analysis. The model enabled transparent assessment and optimisation of net profits and costs associated with four categories of best management practices (cutting edge unproven technologies called ' $A$ ' practice, current best-management practices called ' $\mathrm{B}$ ', common industry or ' $\mathrm{C}$ ' practices, and below industry standards or ' $\mathrm{D}$ ' practice) in the grazing and sugar cane industries. The bioeconomic model was able to solve for RPTs or ERTs assigned to either the entire region or within each of five discrete river basins. Key outcomes from the study were that RPTs could be achieved at an annual cost of $\$ 3 \mathrm{M} /$ year on a whole of region basis. In contrast ERTs could be achieved on a whole of region basis at as net cost of $\$ 16 \mathrm{M} /$ year. ERTs were not able to be feasibly met on a basin by basin basis. This is the first time such a comprehensive and integrated bio-economic model has been constructed for a region within GBR using environmental software that linked available biophysical and economic modelling.
\end{abstract}

Keywords: decision support system, DSS, bio-economic optimisation framework, water quality. 


\section{Introduction}

The Burnett-Mary region in Queensland, Australia includes the southern-most portion of the World Heritage listed Great Barrier Reef (GBR) Marine Park and the Ramsar listed Great Sandy Strait. The region hosts biodiversity values that are globally important. The health of the coastal and inshore marine areas are influenced by the quality and quantity of runoff from five river basins. Increased loads of nitrogen, phosphorus, sediments and pesticides from adjacent catchments have led to chronic changes in environmental conditions for GBR species and ecosystems. To help protect the values of coastal and marine receiving waters, the Burnett Mary Regional Group (BMRG) with funding from the Australian Government commissioned the development of a Water Quality Improvement Plan (WQIP). The focus of the WQIP is to improve water quality through the implementation of agricultural management practices based on the 'ABCD' water quality risk framework for the sugarcane and grazing industries ([1], [2]). 'A' practice represents cutting edge unproven technologies, 'B' represents current best-management practices, ' $C$ ' is common current industry practice and ' $D$ ' is below industry practice expectations. A bio-economic model was developed to assist the Burnett Mary region transparently evaluate the feasibility and costs of various management options to achieve two sets of pollutant load reduction targets, namely Reef Plan Targets (RPTs), the currently formally agreed targets, and more ambitious Ecologically Relevant Targets (ERTs) which might be needed to better protect the values of the GBR. This paper describes the construct and underpinning data used to develop the bio-economic model that explicitly considers the feasibility and net profits/costs of achieving water quality objectives.

\section{The Burnett Mary Region}

The Burnett Mary region in Queensland, Australia covers 56,000 square kilometres (5.6 million ha) of land and encompasses five major river basins (Baffle, Burnett, Burrum, Kolan and Mary). The Baffle, Kolan and the Burnett catchments flow to the GBR Reef Marine Park whilst the Burrum and Mary flow to the Great Sandy Strait Marine Park. The major primary industries are grazing, sugarcane, horticulture, forestry and mining.

\section{Methodology}

The development of the Burnett Mary WQIP involved integrating the outputs from a number of separate supporting projects and biophysical modelling activities across various scales. The approach has built on the outcomes from previous WQIPs, findings of the Scientific Consensus Statement and priorities from Reef Plan 2013 ([4]; [10]; [14]; [15]) and new supporting studies. The key components of this approach have been:

- Use of assumptions and outputs from available science from paddock and catchment scale modelling conducted in Queensland; 
- Coordination and engagement with technical experts and local stakeholders to integrate local knowledge and previous research;

- Financial economic analysis of practices for grazing and sugar cane industries;

- Development and application of a bio-economic modelling framework to assess costs in attaining targets.

\subsection{Data Sources}

Land use data was based on the 2009 land use map derived from the Queensland Land Use Mapping project (QLUMP, [8]). The QLUMP data was used to represent all land uses with the exception of sugar cane. For sugarcane areas, Australian Bureau of Statistics (ABS) data was utilized because the ABS data was widely accepted by stakeholders as better representing the current area of land under sugarcane. Soil data layers were provided by the Queensland Department of Natural Resources and Mines. Stream and gully mapping was provided by the Burnett Mary Regional Group whereas gully and streambank effectiveness assumptions were provided by [16].

\subsection{Landscape Modelling Software}

The Burnett-Mary bio-economic model utilised predictions of catchment streamflow, baseflow and loads (nutrient, sediment and pesticide) derived using Source Catchments [9] and management impacts estimated using paddock scale models. Baseline data was based on a combination of point scale modelling and calibrated catchment scale model outputs. The key components of the linked modelling approach are described below.

\subsubsection{Catchment Model}

The landscape model Source Catchments was used to estimate constituent generation loads and attenuation coefficients for each of the 597 sub-catchment within the Burnett-Mary region. Each sub-catchment was defined based on topography, river basins, land use and the location of existing water quality and flow monitoring stations. Source Catchments modelling from the Paddock to Reef program provides a prediction of end of catchment loads for key pollutants of interest (nitrogen, phosphorus, sediment and pesticides). The variables modelled include: Total Suspended Solids (TSS), Dissolved Inorganic Nitrogen (DIN), PSII Herbicides (PSII), Particulate Nitrogen (PN), Particulate Phosphorus (PP), Dissolved Inorganic Phosphorus (DIP). The 2008-09 baseline results from the Source Catchments modelling were analysed to provide a summary of the relative contribution from the five river basins as a proportion of total load and anthropogenic load [18].

\subsubsection{Paddock-Scale Model}

Farming system models were used to inform sediment and nutrient loads in Source Catchments and to estimate the constituent load reductions associated with various management practices. The APSIM [12] and Howleaky [11] models were used to simulate sugar cane systems, whereas GRASP was used to model grazing systems. 
Output from the farming system models were used to assign constitutive load reductions for each practice class by soil type by management combination representing the various abatement options.

\subsection{Farm Level Financial Cane and Grazing Economics}

Achievement of meaningful water quality targets require practice changes in agriculture. Although infrastructure and all agricultural industries contribute to constituent loads, this study was confined to the sugar cane and grazing industries because of their importance in contributing to water quality issues and the fact that estimates of the contributions of impacts from practice changes could be quantified from modelling assumptions. Financial economic analysis was undertaken for both these industries as part of the WQIP.

The farm level financial economic analysis for sugar cane was conducted for three representative farm sizes (small 75 ha, medium 125 ha, large 250 ha) and two soil types (well drained and less well drained). The analysis included consideration of non-profit related barriers and transaction costs [17].

Similarly the financial economic analysis for grazing in the Burnett Mary region was conducted for three representative farm sizes (small 288 ha, medium 880 ha, large $4134 \mathrm{ha}$ ) and across three land productivity class (high, medium and low; [19]). Both profit and non-profit related financial barriers were also taken into account in the analysis, as outlined in [13].

\subsection{Optimisation Model}

The bioeconomic model components were solved using nonlinear programming with the CONOPT solver in the General Algebraic Modelling System (GAMS) [5]. The model seeks to maximise total net benefits for grazing and sugar cane enterprises as required to meet specified water quality targets at both the regional scale as well as by individual basin. The cost-effectiveness approach adopted in this study, where emissions goals are sought at least cost, is common ([3]; [6]; [7]) because it avoids the difficulty and cost of assessing the benefits associated with improved water quality.

There are a sub-catchments in each catchment labeled $\mathrm{sc}=[1,2 . ., \mathrm{a}]$. The total land area is allocated to $\mathrm{b}$ land use combinations labeled $\mathrm{lu}=[1,2, \ldots, \mathrm{b}]$. These land use combinations contain permutations of sugarcane and grazing BMP options totaling 36 sugarcane management options (18 on good soil and 18 on poor soil) and 90 grazing management permutations (30 options on each of the high, medium and low productivity classes). Three decision variables describe the management options. First, the area allocated to each land use in each sub-catchment denoted by Asc,lu. Second, the intensity with which gully erosion is managed in each sub-catchment denoted by Gsc. Third, the intensity with which streambank erosion is managed in each sub-catchment denoted by Ssc. The intensity of gully, streambank and permanent waterway management is the percentage of the gully system or associated length that is fenced and revegetated. Subscripts eg refers to the length of gullies and es the length of streambanks. Total profit in each catchment is computed: 
$\pi^{n}=$ landuse profit $\mathrm{x}$ landuse area - cost gully management - cost streambank management

$$
=\sum_{s c^{n}=1}^{a^{n}} \sum_{l u^{n}=1}^{b^{n}} \pi^{n}{ }_{s c^{n}, l u^{n}} A^{n}{ }_{s c^{n}, u^{n}}-\sum_{s c^{n}=1}^{a^{n}} \sum_{e g^{n}=1}^{d^{n}} c g^{n}{ }_{s c^{n}, e g^{n}} G^{n}{ }_{s c^{n}, e g^{n}}-\sum_{s c^{n}=1}^{a^{n}} \sum_{e s^{n}=1}^{f^{n}} c S_{s c^{n}, e s^{n}}^{n} S_{s c^{n}, e s^{n}}^{n}
$$

Contaminant loads (TC) are calculated as follows where C refers to either DIN, DON, Particulate N, TN, FRP, DOP, Particulate P, TP, TSS and pesticides (hexazinone, ametryn, atrazine, diuron and tebuthiuron); PP and Gload, Sload refers to exports from gully and streambank respectively:

$$
T C^{n}=\sum_{s c^{n}=1}^{a^{n}} \sum_{l u^{n}=1}^{b^{n}} C_{s c^{n}, u u^{n}}^{n} A_{s c^{n}, l u^{n}}^{n}+\sum_{s c^{n}=1}^{a^{n}} C^{n}{ }_{G L o a d} G^{n}{ }_{s c}+\sum_{s c^{n}=1}^{a^{n}} C^{n}{ }_{S L o a d} S^{n}{ }_{s c}
$$

The optimisation model seeks to maximize profit in each sub-catchment (grazing and sugar cane enterprises only) subject to target emission constraints. The model considered only transition states under the assumption that initial land use areas currently in A and B class practices would not be allowed to be reduced. As such, transitions can only move to an improved state (Figure 1). No land use change (land retirement) was also permitted - solutions could only be derived from practice change. Constraints including (1) farm distributions, (2) soil type, (3) productivity classes, (4) land practice class (A, B, C and D) and (5) land use transitions. Stream bank and gully remediation was also considered with associated opportunity costs.

\subsection{Software Environment}

Source catchment prediction data sets were provided in an Access database. Key data was then exported from the database and manipulated using a Fortran program into a format consistent with the GAM model input data construct. The output results from the GAMS model was post-processed using software developed in Fortran. Outputs included summary text files and raster based gridded data for importing into GIS. Model sensitivity analysis was undertaken using a Fortran wrapper program that systematically modified GAMS input data, initiated the GAMS solver and collated simulation results.

The uniqueness of the developed models is the capacity to

- Consider individual or combinations of constituent abatement targets;

- Consider meeting abatement targets at both the basin or regional scale;

- Evaluate the impact of different levels of funding;

- Estimate the area under A, B, C and D practice and the area transitioned into each of these conditions required to meet abatement targets;

- Estimate the length of streambank and gully remediation. 


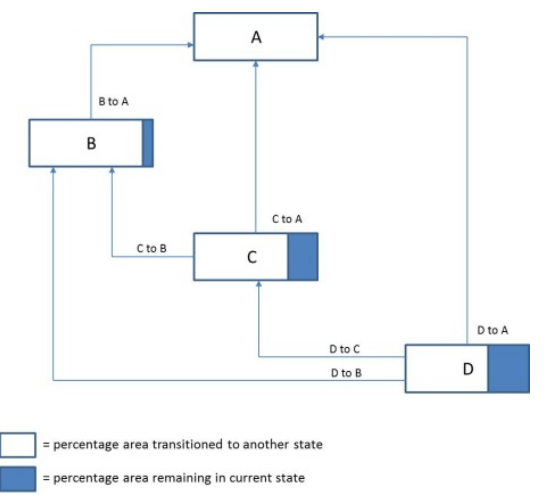

Fig. 1. Schematic of the optimisation model constituent pools

\section{Application}

Numerous targets were evaluated and reported to local stakeholders initially. Two sets of targets were selected as a focus for the WQIP, the Reef Plan Targets (RPTs) and Ecologically Relevant Targets (ERTs). Each target was evaluated at both the regional and individual basin scale and costs to achieve targets were assumed t be over a 20 year period

The RPTs were a $20 \%$ overall reduction in anthropogenic suspended sediment load; a 20\% (based on RPT 2013 target) and 50\% (based on RPT 2009) reduction in anthropogenic loads of particulate nitrogen (PN) and particulate phosphorus (PP); $50 \%$ (based on Reef Plan 2013) reduction in anthropogenic loads of dissolved inorganic nitrogen (DIN); 50\% (based on 'interpreted' Reef Plan 2009) reduction in anthropogenic loads of dissolved inorganic phosphorus (DIP) and 50\% (Reef Plan 2009) and 60\% (Reef Plan 2013) reductions of loads of PSII herbicides (i.e. the 2009 target and the 2013 target respectively).

The ERTs considered in this study are (based on reductions in anthropogenic load from the 2008-09 baseline): $20 \%$ overall reduction in suspended sediment load; a $50 \%$ reduction in particulate nitrogen $(\mathrm{PN})$ and particulate phosphorus (PP); $80 \%$ reduction in dissolved inorganic nitrogen (DIN); $50 \%$ reduction in anthropogenic loads of dissolved inorganic phosphorus (DIP) and $60 \%$ reduction of loads of PSII herbicides. It should be noted that a $50 \%$ reduction in DIP proved to be infeasible and this constituent was limited to a $20 \%$ reduction in the tested scenarios.

\section{$5 \quad$ Results}

\subsection{Costs and Land Use Implications of Achieving Targets}

The annual cost of attaining scenario targets for each river basin are summarised in Table 1, with the associated spatial distribution shown in Figure 2. Figure 3 show the 
spatial patterning of reductions in DIP to meet scenario targets, whereas Figure 4 show the contrasting sediment reduction achievable under limited funding of $\$ 2 \mathrm{M} /$ year and $\$ 8 \mathrm{M} /$ year respectively.

Table 1. Net profit or cost associated with meeting targets in the Burnett Mary region in individual basins or whole of regional scale. Red text indicates a net cost and black text indicates a net profit.

\begin{tabular}{|c|c|c|c|c|c|}
\hline \multirow{2}{*}{ Scenario } & \multicolumn{5}{|c|}{ Annual Cost/Profit (\$M/year) } \\
\cline { 2 - 6 } & Baffle & Kolan & Burnett & Burrum & Mary \\
\hline $\begin{array}{c}\text { 1. Meet RPTs by indi- } \\
\text { vidual basins }\end{array}$ & -1.4 & 0.6 & -4.8 & 0.5 & -2.8 \\
\hline $\begin{array}{c}\text { 2. Whole catchment } \\
\text { RPTs }\end{array}$ & -4.3 & 0.2 & -7.8 & 0.2 & infeasible \\
\hline $\begin{array}{c}\text { 3. Meet all ERTs by } \\
\text { individual basins }\end{array}$ & -5.0 & -16.4 \\
\hline $\begin{array}{c}\text { 4. Whole catchment } \\
\text { ERTs }\end{array}$ & \multicolumn{5}{|c|}{} \\
\hline \begin{tabular}{c} 
ERTs \\
\hline
\end{tabular}
\end{tabular}
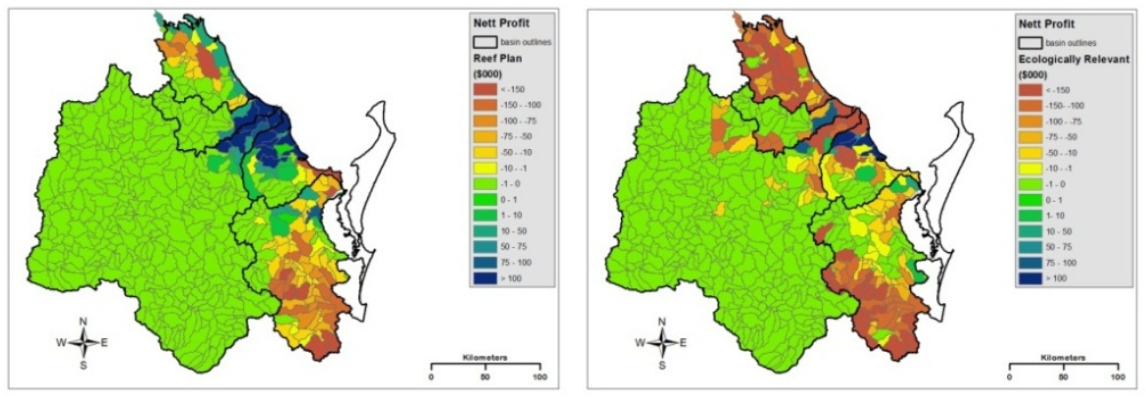

Fig. 2. Net profit associated with the RPTs (left) and the ERTs (right)
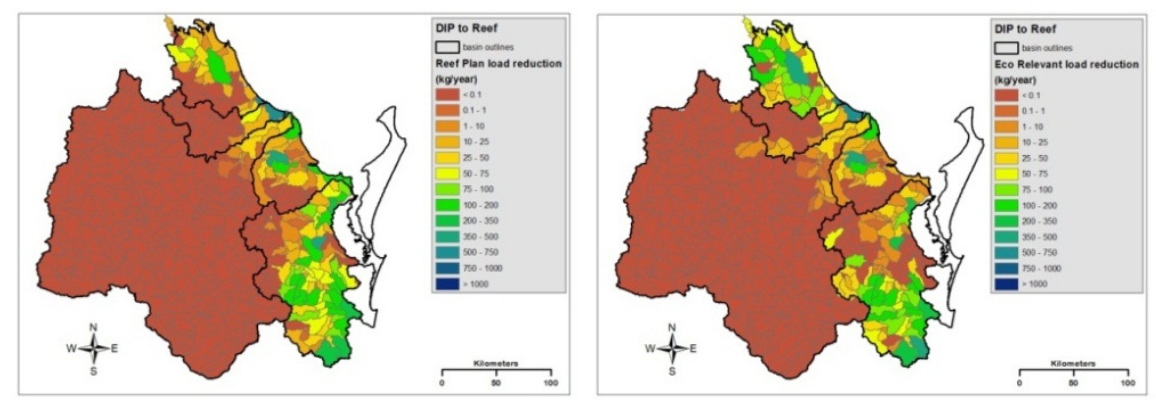

Fig. 3. Reduction in DIP associated with the RPTs (left) and the ERTs (right) 

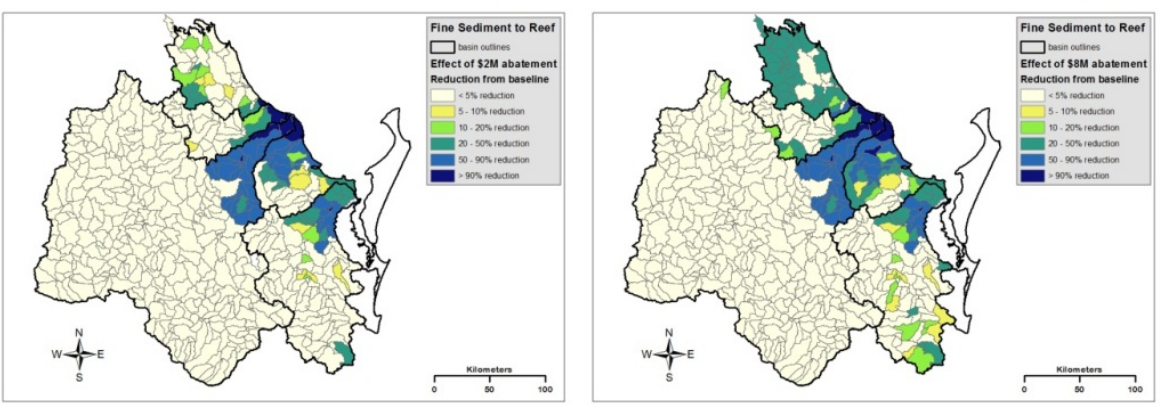

Fig. 4. Reduction in TSS for associated with a $\$ 2 \mathrm{M}$ (left) and $\$ 8 \mathrm{M}$ (right) funding program

\subsection{Land Use Management Changes}

The bioeconomic model predicts that for both RPTs and ERTs in cane all land is required to move to at least B practice to meet whole of basin targets. For RPTs $81 \%$ of cane is required in B practice and $19 \%$ in A practice. For the ERTs the majority of land (over 46,000 ha or $81 \%$ cane) is predicted to be required to be in A practice. The transition in grazing land management is even more challenging, with an additional 131,349 ha required in A practice for RPTs and 243,815 ha to achieve ERTs. For RPTs $19 \%$ of grazing is required in A practice, $51 \%$ in B practice, $22 \%$ in C practice and $7 \%$ in $\mathrm{D}$ practice. In contrast for ERTs, $22 \%$ of grazing is required in $\mathrm{A}$ practice, $49 \%$ in B practice, $21 \%$ in $\mathrm{C}$ practice and $7 \%$ in $\mathrm{D}$ practice.

In the context of available funds (Figure 4), the proportional cane area in practices $\mathrm{A}, \mathrm{B}$ and $\mathrm{C}$ are predicted to be $6 \%, 92 \%$ and $2 \%$ for $\$ 2 \mathrm{M}$ and $14 \%, 84 \%$ and $2 \%$ for $\$ 8 \mathrm{M}$ respectively. A similar transition is predicted for the proportion of grazing areas in practices A, B, C and D which are predicted to be $19 \%, 52 \%, 22 \%$ and $7 \%$ for $\$ 2 \mathrm{M}$ and $22 \%, 51 \%, 21 \%$ and $6 \%$ for $\$ 8 \mathrm{M}$ respectively.

\section{Discussion and Conclusion}

The results for Scenario 1 (meeting the RPTs in each basin, see Table 1) indicate that targets are predicted to be achieved at a modest net profit in the Kolan and Burrum basins. This occurs because of their lesser size compared with the Burnett and Mary and the increased area of sugarcane as a proportion of land use. Net costs are predicted in the Baffle, Burnett and Mary basins because the predictions require practice changes in grazing to achieve the targets, which always incurs a net loss.

If RPTs only have to be met on a whole of region basis, then huge savings can be made (see Scenario 2 Table 1 compared to Scenario 1). The net cost is estimated to be approximately $\$ 3.0$ million/year compared with a net loss of approximately $\$ 7.9$ million/year from Scenario 1.

The difference between the RPTs and the ERTs are in the ambitiousness of the PN and PP targets - only $20 \%$ needs to be met under RPTs compared with $50 \%$ for ERTs. Meeting ERTs poses significant feasibility issues because particulate losses come mostly from grazing land uses, streambank and gully erosion, all of which incur large 
losses. As illustrated in Table 1, ERTs are infeasible to meet in the Mary catchment (Scenario 3). At a whole of region scale, ERTs pose at least an additional \$13.4 million loss/year on agriculture than for RPTs.

Table 1 results also further illustrates that the whole of region targets (Scenarios 2 and 4) are much more cost effective than if targets have to be met on an individual basin level.

This study identified that (1) large and ongoing support will be needed for the grazing industry to achieve sediment and particulate $\mathrm{P}$ and $\mathrm{N}$ targets and (2) the most limiting constituent was DIP. Importantly ERTs cannot be met without substantial costs.

This is the first time such a comprehensive and integrated bio-economic model has been constructed for the study region using environmental software that links available biophysical and economic modelling. The developed framework has a demonstrated capacity to test the effectiveness of alternative land management options, the optimal likely impact from available funding (Figure 4) and the environmental impacts of traditional incentive schemes (such as water way and gully remediation).

Importantly this study has informed the development of the Burnett Mary WQIP. Integration of available science and local knowledge through development of a bioeconomic model shows how net profits/costs of reaching relevant targets $n$ can inform a more realistic implementation plan than previously possible. This is the first time such a comprehensive and integrated bio-economic model has been constructed for a region within GBR using environmental software that linked available biophysical and economic modelling.

Acknowledgements. The authors acknowledge the contributions from Dr Jon Brodie for defining the Ecological Reef Targets, Dr Mark Silburn for paddock scale effectiveness estimates and Dr Robert Ellis and Dr Banti Fentie for the provision of Source Catchments and farm scale modelling results without which the bio-economic model could not have been developed.

\section{References}

1. Anonymous. Paddock to Reef Water Quality Risk Assessment Framework for Sugar Cane. Draft November 2013 (2013a)

2. Anonymous. Reef Plan Water Quality Risk Framework for Grazing. Draft October 2013 (2013b)

3. Beverly, C., Roberts, A., Stott, K., Vigiak, O., Doole, G.: Optimising economic and environmental outcomes: water quality challenges in Corner Inlet Victoria. In: Proceedings of MODSIM 2013, Adelaide (2013), http: / / www .mssanz . org . au /modsim2 013

4. Brodie, J., Waterhouse, J., Schaffelke, B., Kroon, F., Thorburn, P., Rolfe, J., McKenzie, L.: 2013 Scientific Consensus Statement Land use impacts on Great Barrier Reef water quality and ecosystem condition (2013)

5. Brooke, A., Kendrick, D., Meeraus, A., Raman, R.: GAMS-A user's Guide.GAMS Development Corporation, Washington, DC (2008)

6. Doole, G.J.: Cost-effective policies for improving water quality by reducing nitrate emissions from diverse dairy farms: An abatement-cost perspective. Agricultural Water Management 104, 10-20 (2012) 
7. Doole, G.J., Pannell, D.J.: Empirical evaluation of nonpoint pollution policies under agent heterogeneity: regulating intensive dairy production in the Waikato region of New Zealand. Australian Journal of Agricultural and Resource Economics 56, 82-101 (2012)

8. DSITIA, Land use summary 1999-2009: Burnett Mary NRM Region, Queensland Department of Science, Information Technology, Innovation and the Arts, Brisbane (2012)

9. eWater Cooperative Research Centre. Source Catchments User Guide, eWater Cooperative Research Centre, Canberra (2010), ISBN 978-1-921543-29-6

10. Kroon, F., Turner, R., Smith, R., Warne, M., Hunter, H., Bartley, R., Carroll, C.: 2013 Scientific Consensus Statement. In: Sources of sediment, nutrient, pesticides and other pollutants in the Great Barrier Reef, ch. 4 (2013)

11. McClymont, D., Freebairn, D.: Howleaky? Exploring water balance and water quality implications of alternative land uses. A computer program. (C) Dept Natural Resources and Mines, Queensland, Australia (2007)

12. McCown, R.L., Hammer, G.L., Hargreaves, J.N.G., Holzworth, D., Huth, N.I.: APSIM An agricultural production system simulation model for operational research. Mathematics and Computers in Simulation 39, 225-231 (1995)

13. Pannell, D., Roberts, A., Park, G.: Economic analysis of grazing systems for water quality improvement in the Burnett Mary catchment. Report to the Burnett-Mary Regional Group (Natural Decisions Pty. Ltd, February 2014) (2014)

14. Schaffelke, B., Anthony, K., Blake, J., Brodie, J., Collier, C., Devline, M., Warne, M.: 2013 Scientific Consensus Statement. In: Marine and coastal ecosystem impacts, ch. 1, p. 50 (2013)

15. Secretariat Reef Water Quality Plan Protection. Reef Water Quality Protection Plan (2013)

16. Thorburn, P., Wilkinson, S.: Conceptual frameworks for estimating the water quality benefits of improved agricultural management practices in large catchments 180. Agriculture Ecosystems and Environment, 192-209 (2013)

17. Van Grieken, M.E., Pannell, D., Roberts, A.: Economic Analysis of Farming Systems for Water Quality Improvement in the Burnett Mary Catchment. A report prepared for the Burnett Mary Regional Group in cooperation with Natural Decisions. CSIRO Water for a Healthy Country Flagship. Brisbane (January 2014)

18. Waterhouse, J., Maynard, J., Brodie, J., Lewis, S., Petus, C., da silva, E., Mellors, J.: Assessment of the relative risk of degraded water quality to ecosystems of the Burnett Mary Region, Great Barrier Reef DRAFT REPORT A Report for Burnett Mary Regional Group (2014)

19. Whish, G.: GRASP modelling of grazing systems in Great Barrier Reef catchments. Technical Report to Paddock to Reef Integrated Monitoring, Modelling and reporting program funded through the Australian Government's Caring for Our Country Reef Rescue. Department of Agriculture, Fisheries and Forestry, Queensland, Australia (2012) 